\title{
Regulation of Human Megakaryocytopoiesis
}

\author{
MICHAEL W. LONG \\ University of Michigan \\ Department of Pediatrics \\ Division of Hematology/Oncology \\ Room M7510 MSRB I, Box 0684 \\ Ann Arbor, Michigan 48109
}

\section{INTRODUCTION}

A number of in vitro systems are available for the study of both human and murine megakaryocyte development. With these assays, it is possible to cultivate megakaryocyte progenitor cells, ${ }^{1-12}$ immature megakaryocytes, ${ }^{13-15}$ and mature megakaryocytes. ${ }^{16.17}$ However, certain developmental characteristics of this lineage handicap the acquisition of insights into the regulation of megakaryocyte differentiation. Most notably, megakaryocyte progenitor cells are restricted in their proliferative potential generating colonies avcraging $15-25$ cells in size. ${ }^{2,10}$ This reduction in proliferative capacity may be a function of the megakaryocyte's ability to endoreduplicate; i.e., progressively double its DNA content without undergoing cytokinesis. ${ }^{10}$ Until recently, the lack of knowledge concerning factors regulating megakaryocyte development has resulted in colonies of relatively immature cells, further handicapping the usefulness of clonigenic assays in the analysis of megakaryocyte growth and development. Finally, megakaryocyte progenitor cells and early differentiated cells of this lineage (immature megakaryocytes), are heterogeneous cell populations ${ }^{2.13 .18}$ additionally complicating the interpretation of the regulatory mechanisms operative in this lineage.

Information concerning the in vitro requirements for proliferation and differentiation of megakaryocytes suggests that two factors (activities) are required to regulate this process (FlG. 1). Megakaryocyte colony stimulating activity (Mk-CSA) is an obligate requirement for colony formation and primarily affects the proliferative phases of megakaryocyte development, driving the formation of small colonies of immature cells. ${ }^{13,19}$ For optimal colony development (i.e., colonies of large size containing mature megakaryocytes), a second auxiliary factor is required which has little or no role in proliferative events, but stimulates latter phases of differentiation. This distally acting factor (referred to in the murine system as megakaryocyte potentiator activity) lacks the solitary ability to stimulate colony formation, but augments CSA-driven megakaryocyte development by increasing the number of cells per colony, and the size, DNA content, cytoplasmic content, and nuclear complexity of developing megakaryocytes. ${ }^{2,9-12,20,21}$ The recent purification of murine interleukin-3 (IL-3) by Ihle and co-workers ${ }^{22-24}$ represents the first pure molecule to show megakaryocytic colony-stimulating activity, ${ }^{25}$ as well as to support granulocyte and erythrocyte colony development. ${ }^{24.26}$ While this purified hematopoietic growth factor is capable of supporting megakaryocyte colony formation, the colonies formed are typically small in size (containing 10-20 cells), the cells are relatively immature, and the cloning efficiency is low. ${ }^{27}$ Optimal colony formation, in addition to purified IL-3, still requires the addition of an auxiliary activity to enhance colony devclopment. ${ }^{27}$ 
Recent work from this laboratory has shown that tumor promoting phorbol diesters (PDE) substitute for biological sources of this megakaryocyte synergistic activity. ${ }^{2.20}$ In other cell systems, such as the leukemic cell line HL60, or the pheochromocytoma cell line PC12, 4- $\beta$-phorbol 12-myristate 13-acetate (PMA) can induce cell differentiation. ${ }^{28,29}$ Among hematopoietic cells, PMA stimulates granulocyte colony development. ${ }^{30-33}$ In the erythroid lineage these agents stimulate early, burst-forming progenitor cells, ${ }^{34}$ have little effect on later (day 3 ) colony-forming cells ${ }^{34}$ but inhibit terminal phases of erythroid differentiation. ${ }^{34,35}$ Data from our laboratory indicates that tumor-producing phorbol diesters stimulate both megakaryocyte progenitor cells and the differentiation of immature megakaryocytes. 2,20

The development of in vitro assays for human megakaryocyte development lags behind the murine studies, undoubtedly due to the relative scarceness of this resource (human bone marrow) and further technical difficulties in growing megakaryocytes. Hu-

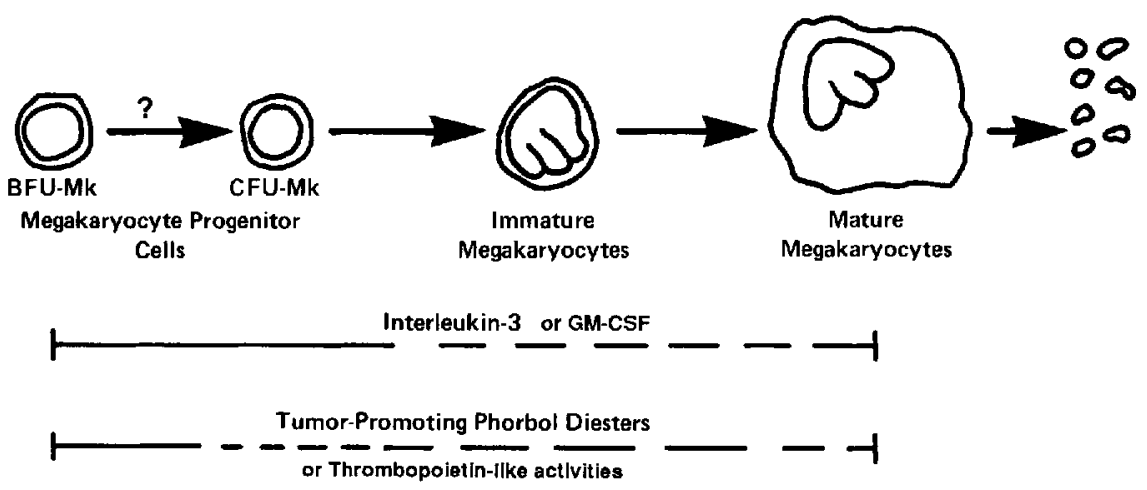

FIGURE 1. Regulation of megakaryocytopoiesis. The cellular elements of this lineage are: the burst-forming progenitor cell (BFU-Mk), an early progenitor which antedates the more mature colony forming cell (CFU-Mk); ${ }^{2,27}$ the immature megakaryocyte, a lymphoid-size cell expressing megakaryocyte specific antigens, or enzymes and having a high nucleus:cytoplasm ratio ${ }^{4,13,18,20}$ and the casily recognizable mature megakaryocyte. Factors regulating the development of these cells are IL-3 and GM-CSF as well as thrombopoietin-like synergistic co-regulators (see text). Solid lines indicate obligate requirement for growth factor, broken lines dual regulation. ${ }^{2,25: 27}$

man megakaryocytes, for example, require large concentrations of plasma for growth, ${ }^{36,37}$ and fetal calf sera is often inhibitory. ${ }^{36,38}$ Nonetheless, human megakaryocyte progenitor cells can now be grown in a variety of semisolid support systems: plasma clot, ${ }^{36,37}$ methylcellulose, ${ }^{36,37}$ and agar. ${ }^{21}$ Such studies report various positive and negative regulatory influences in vitro. However, large differences in stimuli, kinetics of development, and cloning efficiency are reported and systematic comparison of culture conditions and stimuli are lacking.

In regards to the factors regulating human megakaryocytopoiesis, a number of studies have shown circulating Mk-CSA in disorders such as aplastic anemia ${ }^{3,37}$ or amegakaryocytic thrombocytopenia. ${ }^{\prime}$ Other investigations have indicated that disorders such as reactive thrombocytosis, or primary thrombocythemia, lack detectable Mk-CSA and suggest that these disorders may involve an auxiliary factor. ${ }^{1,39}$ Thus, these clinical data provide a powerful rationale for examination of these plasma activities (and similar activities from $\mathrm{CM}$ ) in human megakaryocyte development. 
We report that the optimal in vitro development of human megakaryocytes requircs two synergistic regulatory activities; megakaryocyte colony-stimulating activity is an obligate requirement for megakaryocyte proliferation and stimulates the formation of small colonies of immature megakaryocytes; optimal megakaryocyte development requires an auxiliary activity which influences megakaryocyte size, cytoplasmic content, antigcnic expression, and maturational stage.

\section{MATERIALS AND METHODS}

\section{Preparation of Bone Marrow Cells}

Following informed consent, bone marrow aspirates arc obtained from the posterior iliac crest of normal volunteers. Volunteers were reportedly free of pharmaceuticals for three weeks prior to the aspiration.

Bone marrow cells are aspirated into syringes containing $100 \mathrm{U}$ of preservative-free sodium heparin (University of Michigan) in normal saline. In order to prevent peripheral blood dilution, aspirate volumes were kept at $0.5-0.8 \mathrm{ml}$. Aspirates were immediately mixed with $100 \mu \mathrm{L}$ of McCoy's 5A medium (see below) containing 100-125 U DNase I (Sigma, St. Louis, MO). The addition of DNase to the samples improves the recovery of cells following density separation. The aspirate is diluted with McCoy's $5 \mathrm{~A}$ medium (Gibco, Grand Island, NY), layered over ficoll/diatrizoate (density $=1.077 \mathrm{gcm}^{-3}$, Histopaque, Sigma Chemical Co., St. Louis MO) and spun for $20 \mathrm{~min}$ at $400 \mathrm{~g}$. The interface cells are collected, washed $\times 2$, and subjected to two rounds of plastic adhercnce. For adherence, low density marrow cells are incubated $\left(3 \times 10^{5} / \mathrm{ml}\right)$ in McCoy's $5 \mathrm{~A}$ medium containing $5 \% \mathrm{FCS}$ for one hour at $37^{\circ} \mathrm{C}$, after which the suspension cells are removed and subjected to a second adherence step. The resultant nonadherent, lowadherent cells (NALD; $\leq 0.1 \%$ macrophages) are used as target cells in all experiments.

\section{Hematopoietic Progenitor Cell Assays}

Progenitor cells are cultured in a modification of a previously described agar system. ${ }^{2,20}$ These cultures are performed as described for murine cells, ${ }^{2,20}$ except that $30 \%$ human plasma replaces the FCS and $\mathrm{PGE}_{2}$ is omitted. Briefly, NALD cells are cultured in supplemented McCoy's 5A medium containing $30 \%$ heparinized human plasma and $0.25 \%$ bactoagar (Difco, Detroit, MI). Bone marrow cells are co-cultivated with conditioned media from a human carcinoma cell line, human lung cell lines or PHA-LCM (vide infra). Incubation conditions are identical to those for methylcellulose cultures. Unless otherwise stated, all cultures are plated at a limiting cell density of $5 \times$ $10^{4} \mathrm{NALD}$ cells $/ \mathrm{ml}$ (the CFU-Mk assay, in agar, is linear over a range of $25-100 \times 10^{3}$ cells $/ \mathrm{ml}, \mathrm{r}=0.95, \mathrm{r}^{2}=0.90, \mathrm{y}$ intercept $=-0.3$ ).

Megakaryocyte colonics are identified by their morphological characteristics as described elsewhere ${ }^{36-38}$ Morphological identification of megakaryocyte colonies (as presented in Results) was validated by immunoperoxidase labeling using platelet-megakaryocyte specific antibodies (see below). Individual marrow sample replicate cultures were air dried $^{7}$ and stained by immunoperoxidase labeling. These parallel culturcs gave equivalent results to those based on morphological identification for each of the regulatory activities examined (data not shown).

The sample number reported ( $\mathrm{n}$ ) represents the number of individual marrows examined. For each marrow, all cxperimental conditions are cultured as 3-5 replicate cultures/ condition. Valucs are expressed as mean \pm SD of the mean replicate culture values averaged over the indicated number ( $\mathrm{n}$ ) of individual marrow samples. 


\section{Conditioned Media}

Various conditioned media are used as sources of hematopoietic regulatory activities. These are: PHA-LCM, a standard source of such regulatory activities, ${ }^{36.37}$ and media conditioned by 3 human cell lines: a bladder carcinoma cell line, 5637 (a generous gift of the late Dr. Jorgen Flogh, Sloan Kettering Institute, Rye, NY), and 2 lung cell lines (A549 and Hel 299 from American Type Tissue Culture Collection, Rockville, MD).

\section{Human Phytohemagglutinin Stimulated Leukocyte Conditioned Medium (PHA-LCM)}

Human leukocyte-rich plasma is obtained by admixing peripheral blood (buffy coat) with $2 \%$ methylcellulose $(1: 2)$ and the leukocyte-rich plasma collected 30-60 min later. The cells are washed $3 \times$ with IMDM, and cultured $\left(1 \times 10^{6} / \mathrm{ml}\right)$ in IMDM containing $10 \%$ FCS and 1\% PHA (Welcome Diagnostics, Greenville, NC). After 7 days, conditioned medium is harvested by centrifugation, filter sterilized $(0.22 \mu)$ and stored at $-80^{\circ} \mathrm{C}$ until used.

\section{Human Lung Conditioned Medium (HLuCM)}

Human lung cells are grown in RPMI 1640 and containing 10\% FCS (Hyclone, Ogden, UT) supplemented with $1.0 \%$ essential amino $(50 \times), 0.75 \%$ nonessential amino acids $(100 \times)$ and $0.75 \%$ vitamins $(100 \times)$, v/v (all from Gibco), $7.5 \%$ sodium bicarbonate, and $0.25 \%$ glucose. These cells are grown to confluence, and the medium conditioned for 5 days. Supernatant medium is decanted, centrifuged, filter sterilized and stored at $-80^{\circ} \mathrm{C}$.

\section{Human Bladder Carcinoma (5637) Conditioned Medium (BlCM)}

Adherent human bladder carcinoma cells are grown in RPMI 1640 containing $0.75 \%$ $(\mathrm{w} / \mathrm{v})$ glutamine $(100 \times, \mathrm{Gibco})$ and $2 \% \mathrm{FCS}$. Cells are cultured in $75-\mathrm{cm}^{2}$ flasks on Cytodex -3 beads (Pharmacia, Piscataway, NJ). Cells are grown to confluence (on beads), and the medium conditioned for 3-4 days. Following centrifugation, the conditioned medium is concentrated 5 -fold by ultrafiltration (Amicon YM-10 membrane, Amicon Corp., Lexington, MA), filter sterilized, and stored at $-80^{\circ} \mathrm{C}$.

Optimal concentrations of these conditioned media are determined using reciprocal titrations as reported elsewhere. ${ }^{14}$ These CMs are examined over a range of $0.1-30 \%$. Each CM is titrated alone, and in the presence of the putative, synergistic regulator. The addition of known synergistic activities (e.g., PMA) serves to unmask relatively weak CSAs. These concentration:responsiveness studies thus detect differential thresholds (either by examining broad ranges of $\mathrm{CM}$ alone, or by co-culture) or inhibitors (by dilution). However, such studies cannot exclude the presence of an inhibitory activity which has an identical response curve in both types of titration experiment. While improbable, such an event may reduce or mask lineage specific or nonspecific activities. The concentrations routinely used are: $\mathrm{BICM}, 7.5 \%$, HLuCM, 10\%, PHA-LCM, $10 \%$ (final concentrations).

\section{Tumor-Promoting Phorbol Diesters}

Phorbol diesters (Sigma) are prepared as stock solutions of $10^{-5} \mathrm{M}$ in dimethylsulfoxide and kept in the dark at $-20^{\circ} \mathrm{C}$ until used. Fresh stock solutions are prepared every 
3 weeks. Immediately before use, a working solution is made using culture medium as the diluent.

\section{Human Plasma Samples}

Plasma is obtained from normal volunteers following informed consent. Plasma is anticoagulated with preservative-free heparin and immediately spun $(1500 \mathrm{~g} \times 20 \mathrm{~min})$ to produce platelet poor plasma. This plasma is filter sterilized $(0.22 \mu)$ and used immediately. Normal plasma, alone, does not stimulate colony development, but is screened for the optimal concentration supporting colony growth $(30-40 \%$, final concentration).

\section{Liquid Suspension Cultures}

Bone marrow NALD cells are prepared as described above. Following plastic-adherence, the cells are subjected to nylon wool adherence to remove residual adherent cells and B lymphocytes. ${ }^{20}$ These human marrow NALD-B cells are cultivated $\left(1-2 \times 10^{5} / \mathrm{ml}\right)$ in the presence of regulatory activities for 5 days. Culture conditions are as described above.

\section{Human Recombinant Hematopoietic Growth Factors}

Recombinant human IL-3, GM-CSF and IL-6 were the generous gift of Dr. Steven Clark, Genetics Institute, Cambridge, MA. The rh-IL-3 (lot 1039-86; sp. act 0.5-1.0 X $10^{7} \mathrm{U} / \mathrm{mg}$ ) is E. coli expressed and 99\% pure. Human, rh-GM-CSF (lot CD19D2-701; sp. act. $1 \times 10^{7} \mathrm{U} / \mathrm{mg}$ ) is $E$. coli expressed and $99 \%$ pure.

\section{Solid-Phase Radioimmunoassay (SPRIA) for Hematopoietic Cell Antigens}

In order to rapidly and precisely define factor-related changes in megakaryocyte development, we developed a SPRIA to detect megakaryocyte antigens. ${ }^{40}$ Briefly, the SPRIA uses a filtration membrane as a solid support (Durapore, Millipore Corp., Bedford, MA) and antibody-bound ${ }^{125}$ I-staphylococcal protein A (SPA, ICN, Irvine, CA) as a detection system. Intact, cultivated bone marrow cells (NALD-B) are loaded into microtiter wells, and all washes and incubations performed in situ. Antibody binding is thus performed in a solid-phase, i.e., antigens remain membrane associated on intact cells which are affixed to the polyvinylidene membrane. Additionally, this procedure prevents cell loss due to repetitive centrifugation, and wash steps.

\section{Antibody Specificity}

Three platelet/megakaryocyte specific antibodies are used in these studies. ${ }^{40}$ These were: Anti-Factor VIIIag (Dako, Santa Barbara, CA), a monospecific heterologous antibody adsorbed $3 \times$ with human WBCs, RBCs, and mouse liver powder; and two monoclonal antibodies: antiplatelet glycoprotein IIb/IIIa (a generous gift of Dr. Robb Todd, University of Michigan), and antiplatelet glycoprotein Ib (Dako). The final concentration of each antibody was: anti-Factor VIIIag 1:1000, anti-GP IIb/IIIa 1:30, and anti-Ib 1:20. In order to improve detection, a cocktail of these antibodies (each at optimal concentra- 
tion) was used. All results reported are based on the signal detected using this antibody cocktail.

\section{Megakaryocyte Morphology}

Megakaryocytes are identified using avidin-biotin immunoperoxidase conjugates ${ }^{41}$ to label megakaryocyte colonies in situ or cytocentrifuge preparations of cells grown in suspension. Analysis of bone marrow cellular reactivity using these antibodies by immunocytochemistry shows only mature and immature megakaryocyte positive for the peroxidase reaction. ${ }^{40}$ As well, antibody specificity in the RIA system was examined by using purified human PMNs, HL60 cells, and input NALD cells. None of these latter controls shows specific binding differing from zero.

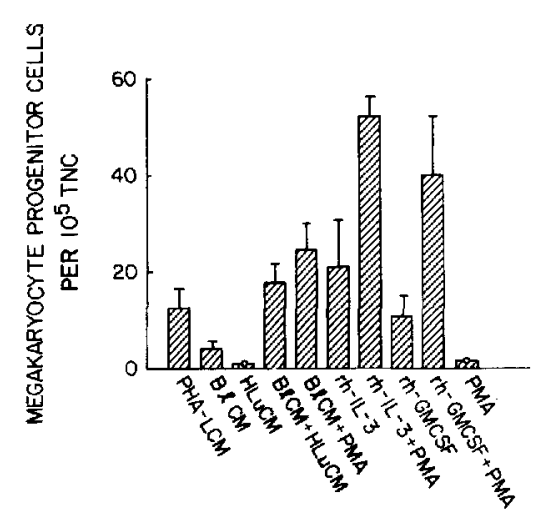

FIGURE 2. Responsiveness of human megakaryocyte progenitor cells to recombinant growth factors and various conditioned media. Human progenitor cells cultured in agar as described in Methods. Values are mean \pm SD of 10 individual marrow samples cultured at 3-5 replicate cultures per condition, BlCM, human bladder cell line 5637 CM $(7.5 \%$ final concentration); PMA, phorbol myristate acetate; PHA-LCM, phytohemagglutinin stimulated human leukocyte conditioned medium (7.5-10\% v/v, final concentration); HLuCM, human lung conditioned medium; rh-IL-3, recombinant human interleukin-3 tested at $1 \mathrm{ng} / \mathrm{ml}$; rh-GMCSF, recombinant granulocyte/macrophage CSF tested at $300 \mathrm{ng} / \mathrm{ml}$. Target cells are NALD-B cells. High concentrations $(20 \%$ v/v of HLuCM stimulate 7-10 granulocyte clusters/10 $0^{5}$ TNC; contrasts of $\mathrm{BlCM}$ vs BlCM plus HLuCM or PMA or rh-IL-3 versus rh-IL-3 plus PMA are significant ( $p \leq 0.05$, Student $t$ test). Bars marked with a zero show no colony development.

\section{RESULTS}

\section{Human Megakaryocyte Colony Development In Vitro}

In vitro human megakaryocyte colony development requires at least one regulatory activity: an obligate megakaryocyte colony-stimulatory activity necessary for progenitor cell maintenance and proliferation. Conditioned medium both from the human bladder carcinoma line 5637 (BICM) and from PHA-LCM are required for colony formation but support limited proliferation of bone marrow megakaryocyte progenitor cells (CFC-Mk) (FIG. 2). These conditioned media were active in final concentrations of $2.5-20 \%$ (optimal $=7.5 \%$ ) and support $5-12$ CFC-Mk $/ 10^{5}$ NALD-B cells. Colonies stimulated by optimal concentrations of these CMs contained 3-20 megakaryocytes of relatively small size.

Conditioned medium from the human lung cell line A549 (HLuCM) lacks the solitary ability to promote CFU-Mk growth. However, co-culture of HLuCM with conditioned medium from 5637 bladder carcinoma cells significantly augments megakaryocyte colony formation resulting in $17.6 \pm 7 / 10^{5} \mathrm{TNC}$ megakaryocyte progenitor cells contrasted to $3.9 \pm 3$ colonies formed in the presence of $\mathrm{B} 1 \mathrm{CM}$ alone $(\mathrm{n}=10, p \leq 0.05$, Student $t$ test) 
(FiG. 2). Megakaryocyte colonies grown in the presence of these two conditioned media contain 3-200 megakaryocytes which were larger than megakaryocytes stimulated with bladder carcinoma conditioned medium alone (not shown).

In order to identify recombinant hematopoietic factors capable of regulating megakaryocyte development, we next examined recombinant human IL-3 and GM-CSF for solitary ability to stimulate megakaryocyte colony development or for their actions in augmenting CSF-driven colony formation. Interleukin-3 alone supports equivalent colony formation to that seen with PHA-LCM or BICM (FIG. 2). Nonetheless, addition of PMA to IL-3 containing cultures significantly augments megakaryocyte colony formations yielding $52 \pm 4$ colonies $/ 10^{5}$ TNC. This augmentation megakaryocyte colony formation occurs over an IL-3 concentration range of $100-1000 \mathrm{pg} / \mathrm{ml}$ (data not shown). Similarly, granulocyte/macrophage CSF stimulated equivalent megakaryocyte colony formation but requires an approximate 100 -fold increase in concentration (FIG. 2). Synergistic interactions also occur between GM-CSF and phorbol diesters. At $300 \mathrm{ng} / \mathrm{ml}$ GM-CSF, approximately 4 megakaryocyte colonies are developed $/ 10^{5} \mathrm{TNC}$, whereas $10^{-8} \mathrm{M}$ PMA yields $48 \pm 23$ colonies (mean $\pm \mathrm{SD} ; \mathrm{n}=4$ ). Additionally, recombinant erythropoietin, IL-1, or IL-6, do not show solitary colony-stimulating activity or the ability to synergize with sources of Mk-CSA such as interleukin-3 (data not shown).

The requirement for two synergistic activities is confirmed in studies on the effects of phorbol diesters on human megakaryocyte colony development. Phorbol myristate acetate can substitute for HLuCM (but not for (BICM), a source of Mk-CSA) and increase megakaryocyte colony numbers. In the presence of a source of Mk-CSA, PMA potentiates megakaryocyte colony development over a range of $10^{-9}-10^{-7} \mathrm{M}$ (FIG. 3A). Addition of $10^{-8}$ PMA to optimal amounts of Mk-CSA causes a 9-fold increase in megakaryocyte colony formation. Examination of the structure activity relationship of phorbol diesters indicates that the tumor-promoting phorbol diesters PMA and phorbol dibutyrate (PdBU) are capable of substituting for the biological source of the synergistic activity whereas the inactive parent compound phorbol, or the vehicle DMSO are not (FIG. 3B).

In order to precisely identify the biological effects of the synergistic activity observed in human lung conditioned medium, human low-density nonadherent cells were cultivated in suspension culture in the presence of sources of Mk-CSA alone or co-cultivated with this activity and either PMA or a source of the biological stimulatory activity (human lung conditioned medium).

Morphological analysis of megakaryocytes grown in the suspension phase cultures indicates a substantial increase in the percentage of mature megakaryocytes stimulated by the addition of secondary synergistic activity (FIG. 4). When cultivated in the presence of Mk-CSA alone, only immature megakaryocytes are seen. These cells have a high nucleus: cytoplasm ratio, are small in size and contain low amounts of megakaryocyte antigen (as visualized by immunolocalization). The addition of either source of synergistic co-regulator results in the reversal of the nucleus:cytoplasm ratio, an increase in size, increased nuclear lobulation, and a visual increase in the content of megakaryocyte specific antigens per cell.

The obscrvation of increased megakaryocyte antigen content suggests that the synergistic co-regulator is capable of regulating megakaryocyte antigenic expression. Two alternative approaches indicate that one of the biological roles of the synergistic coregulator is the regulation of megakaryocyte antigens. Suspension phase cultures of nonadherent low-density (NALD-B) cells, incubated in the presence of Mk-CSA alone or co-incubated with Mk-CSA and human lung conditioned medium increase the number of antigen positive cells. Megakaryocyte-CSA alone is capable of increasing the number of antigen positive cells roughly four-fold over input levels (FIG. 5) The addition of optimal concentrations $(2.5 \%)$ of human lung conditioned medium doubles of the frequency of antigen positive megakaryocytes contrasted with cells cultivated in Mk-CSA alone. 
In order to more precisely quantitate the regulation of megakaryocyte antigenic content, we developed a solid phase radioimmunoassay (SPRIA) capable of detecting as few as 300 megakaryocytes. ${ }^{40}$ This SPRIA indicates that Mk-CSA alone stimulates low amounts of antigen expression, whereas co-cultivation of Mk-CSA and $10^{-8}$ M PMA results in a 14-fold increase in specific binding compared to unstimulated cells (TABLE 1).

\section{DISCUSSION}

Optimal human megakaryocyte development in vitro requires the presence of at least two regulators which promote proliferation and differentiation of these cells. Conditioned

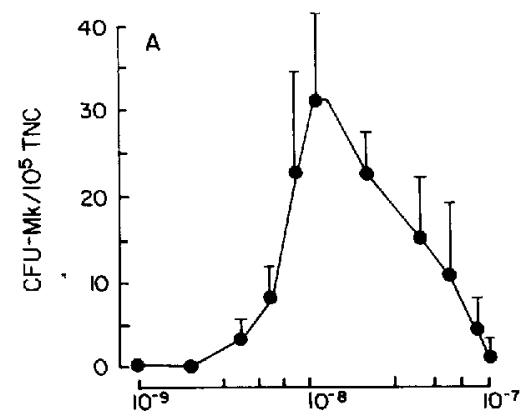

CONCENTRATION OF PMA

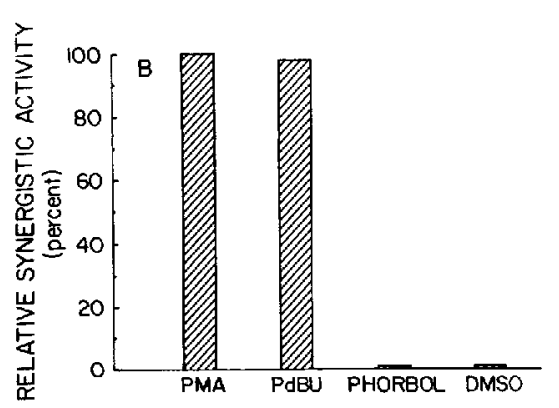

FIGURE 3. (A) Responsiveness of human megakaryocyte progenitor cells to phorbol myristate acetate. Progenitor cell assays performed as in text. All assays contain optimal concentrations $(7.5 \% \mathrm{v} / \mathrm{v}) \mathrm{BICM}$. Values are mean $\pm \mathrm{SD}(\mathrm{n}=$ 4). (B) Structure-activity relationship between various phorbol esters and megakaryocyte-potentiating ability. For comparison, the maximum synergistic activity of $10^{-8} \mathrm{M}$ PMA, when co-cultured with optimal amounts of BICM $(7.5 \%$ final concentration) is expressed as 100 . All phorbol esters tested at $10^{-8} \mathrm{M}$. Values are means of 4 individual marrow samples each cultured at 3-5 replicate culture/experiment/condition. Optimal value of BlCM + PMA represents $24.6 \pm 11.3$ CFU-Mk $10^{5}$ TNC. PMA, phorbol 12-myristate 13-acetate, PdBU, phorbol 12, 13-dibutyrate; PHORBOL, the parent alcohol of PMA; DMSO, dimethylsulfoxide.

medium from bladder carcinoma cells (line 5637), peripheral blood leukocytes (PHALCM), or the recombinant growth factors, IL-3 or GM-CSF, supports limited megakaryocyte development generating colonies of immature megakaryocytes. Colony development is greatly enhanced by the addition of conditioned medium from a neoplastic lung cell line (A549), or tumor promoting phorbol diesters suggesting the existence of dual or multiple regulators in this process. The synergistic co-regulator lacks the solitary ability to stimulate progenitor cell proliferation, but appears to amplify megakaryocyte maturation by increasing the number and size of developing megakaryocytes, the expression of antigenic determinants, and cytoplasmic and nuclear maturation.

We examined the actions of recombinant human hematopoietic growth factors to precisely establish which factors in the complex condition media may control mega- 
karyocyte proliferation. Both rh-IL-3 and GM-CSF support the proliferation of megakaryocyte progenitor cells but have limited effects on maturation. Such observations extend and confirm recent reports by Quesenberry and co-workers showing that murine rh-IL-3 and GM-CSF support megakaryocyte colony formation. ${ }^{25}$ While human megakaryocyte colony numbers are higher in the presence of recombinant growth factors than conditioned media, colony development is suboptimal unless another regulatory factor is present. Such an activity is found in HLuCM and its substitute PMA. The addition of phorbol diesters to recombinant growth factors results in similar increases in colony number and size as was observed with the biological activity present in human lung

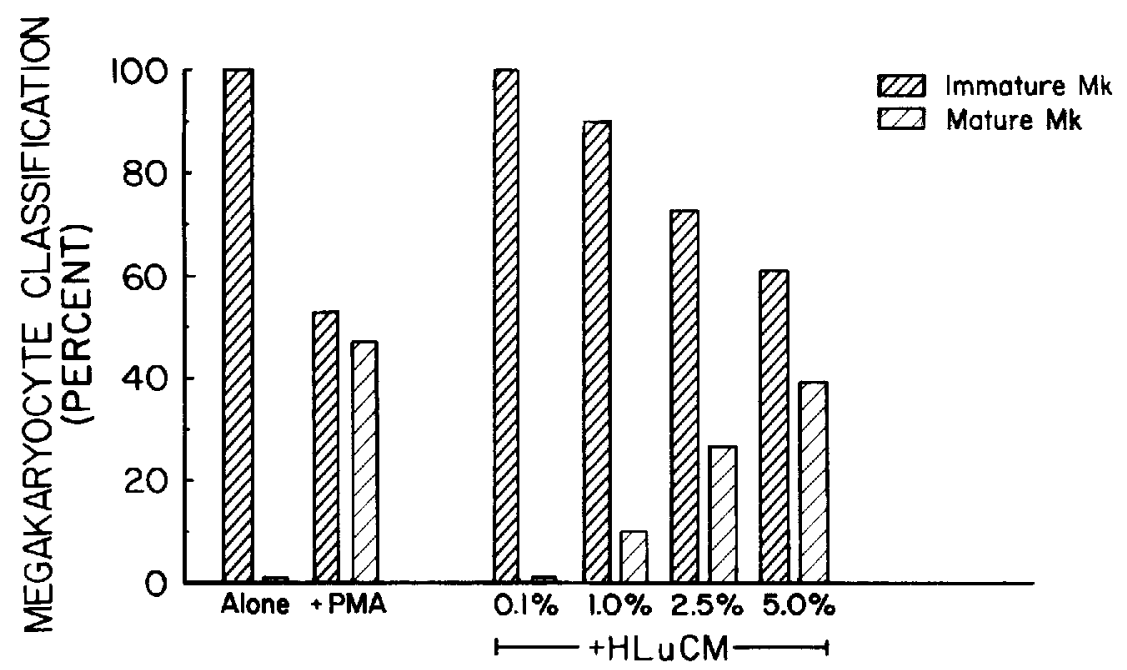

MK - CSA CONTAINING CULTURES

FIGURE 4. Morphological changes in suspension phase megakaryocytopoiesis. Megakaryocytes grown in suspension phase cultures as in Methods. Quantitative values are mean $\pm \mathrm{SD}(\mathrm{n}=3)$ except for BICM + HLuCM titration which are 5 separate cultures from an individual marrow donor. Abbreviations as in FIGURE 2. Megakaryocytes are identified by immunoperoxidase and classified as: immature cells $\leq 18$ diameter, high nucleus:cytoplasm content ${ }^{18}$ and low amounts of antigen (trace $-1+$ ), and mature cells being $>20$ diameter, low nucleus:cytoplasm ratio and large amounts of antigen $(++$ to ++++ ); for classification approximately 100 megakaryocytes (mature and immature) were counted per condition/donor. Percentages are amount ( $v / v)$ of HLuCM added to a constant concentration of BICM $(7.5 \%, \mathrm{v} / \mathrm{v})$.

conditioned medium. Thus the use of recombinant growth factors plus PMA as a substitute for the biological co-regulator allows unambiguous confirmation of the need for two factors in megakaryocyte development.

The observed increases in megakaryocyte antigenic expression also substantiates our observations of synergistic regulation of megakaryocyte maturation. In suspension cultures, both HLuCM and PMA elevate total antigenic expression over that seen with Mk-CSA alone. Notably, the number of total antigen positive cells (i.e., immature plus mature megakaryocytes) increases greatly. The addition of Mk-CSA alone results in a 4-fold increase in megakaryocyte numbers over input levels. Co-culture of Mk-CSA with 


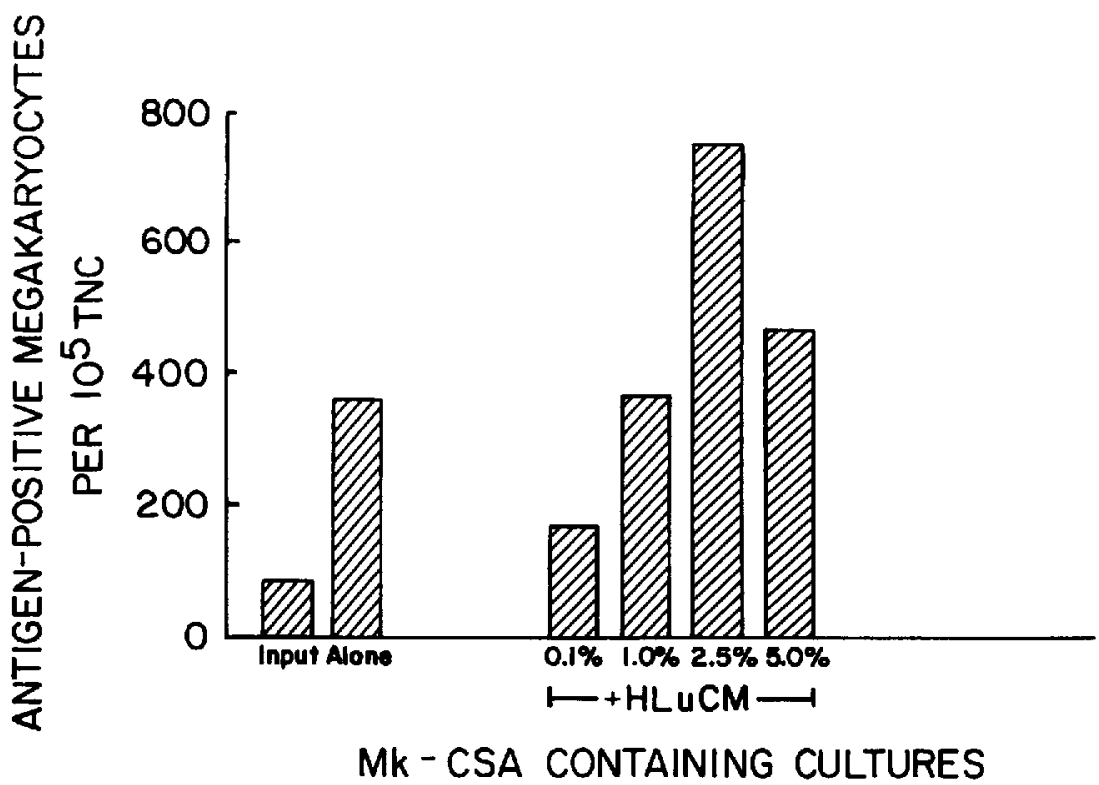

FIGURE 5. Numerical changes in suspension phase magakaryocytopoiesis. Cultures and abbreviations as in FIGURE 4.

HLuCM yields a 9-fold increase in megakaryocyte numbers. Finally, substitution of PMA for HLuCM causes a 37-fold increase (Long et al., unpublished observations). The increases observed in both colony numbers and the total number of antigenic positive megakaryocytes suggest that synergistic regulator(s) either stimulate proliferation, without supporting colony formation, or, alternatively, regulate the expression of megakaryocyte antigens making previously unrecognizable cells antigen positive. This latter hypothesis was confirmed in SPRIA studies showing that antigen positivity per cell increases in the presence of Mk-CSA and the synergistic co-regulator. However, these studies do not allow distinction between actions on the progenitor cell, which is capable of proliferation, or antigen negative immature megakaryocytes, which then become antigen positive; both target cell populations exist in these cultures. ${ }^{16}$

TABLE 1.

Regulation of the Antigenic Content of Developing Megakaryocytes ${ }^{a}$

\begin{tabular}{lc}
\hline Stimuli & Specific Binding ${ }^{125}$ I-SPA (pg/10 ${ }^{5}$ TNC) \\
\hline Input & 2.0 \\
After incubation $^{b}$ & $20.7 \pm 5$ \\
Mk-CSA $^{c}$ & $45.7 \pm 24$ \\
Mk-CSA $^{-P_{M A}}{ }^{d}$ & $350.0 \pm 16$ \\
\hline
\end{tabular}

${ }^{a}$ Solid-phase radioimmunoassay (SPRIA) performed as in Methods. Stimulation of antigenic content after 5 days of culture. Values are mean $=S D(n=11$, except for input, which is a single sample). Values are means of triplicate SPRIA determinations for each donor.

${ }^{b}$ Incubation of unstimulated NALD-B cells in the presence of bovine serum albumin (BSA).

${ }^{C} \mathrm{Mk}=\mathrm{CSA}$, megakaryocyte colony-stimulating activity.

${ }^{d}$ PMA, phorbol myristate acetate. 
Two-tiered regulation of human megakaryocyte development was first described by Harker and Finch who proposed that separate activities regulate the expansion versus the size of developing megakaryocytes ${ }^{42}$ In the murine system, early after thrombocytopenia, megakaryocytes increase in size, cytoplasmic content, and maturity. ${ }^{43-46}$ With prolonged stimulation changes are seen in megakaryocyte number. ${ }^{47,48}$ These observations suggest that two phases of in vivo regulation occur, an early thrombopoietin response affecting megakaryocyte/platelet development, and a later Mk-CSA responsive phase affecting megakaryocyte number (proliferation).

In vitro, Hoffman and co-workers observe that purified Mk-CSA acts at the level of the megakaryocyte progenitor cell stimulating the formation of colonies of small immaturc megakaryocytes. ${ }^{19}$ They further suggest that activities such as thrombopoietin regulate more distal events. Consistent with this, Gewirtz et al. failed to detect Mk-CSA in patients with reactive thrombocytosis and hypothesized the presence of a second regulator in these patients. ${ }^{39}$ We show that a synergistic co-regulator functions with Mk-CSA to regulate megakaryocyte size, cytoplasmic content, and antigen maturity. Thus, the in vitro actions of this synergistic regulator mimic some of the known in vivo effects of thrombopoietin. Discovering whether these regulators are different molecules or members of the same family of regulators must await purification of both factors.

The eventual purification of an activity regulating the latter phases of megakaryocyte development will allow functional comparison between this and other factors influencing differentiation. Rosenberg and co-workers recently purified a protein, megakaryocyte stimulatory factor (MSF), having the ability to stimulate platelet factor 4 synthesis, a functional characteristic associated with megakaryocyte maturation. ${ }^{49.50}$ It is premature to establish identity between this molecule and the activity in HLuCM. While the latter is not purified, the similarities are that both influence megakaryocyte maturation. However, the activity in HLuCM works at the progenitor level to augment CSA-driven colony formation, stimulates increases in sizc, cytoplasmic content, nuclear complexity, and antigenic content in developing megakaryocytes. These functions were not examined for MSF (except that it is not a CSA). Further work at the biochemical level (for our activity) and the cellular level (for MSF) is required to identify similarities or co-factor type interactions between these factors.

We conclude that optimal human megakaryocyte development requires the presence of two synergistic regulatory activities. Regulation of megakaryocyte proliferation is an activity of IL-3 or GM-CSF, either of which is obligate for colony formation and stimulates proliferation and limited differentiation of megakaryocytes. Full megakaryocyte development requires a synergistic co-regulator which, lacking CSA, influences characteristics of megakaryocyte maturation such as: number, size, cytoplasmic content, antigenic content, and maturity. However, some of the studies here utilize conditioned medium rather than purified proteins, and accessory cell-depleted bone marrow rather than purified cells, making direct assessment of the role of either factor and their target cells difficult. Thus it cannot be concluded that accessory cells are not involved in these studies or that more than one synergistic regulator is present. Given these limitations, further characterization of the role of co-regulator and its targets must await purification of this synergistic regulatory factor and the use of purified target cell populations.

\section{REFERENCES}

1. Hoffman, R., E. Mazur, E. Bruno \& V. Floyd. 1981. Assay of an activity in the serum of patients with disorders of thrombopoiesis that stimulates formation of megakaryocytic colonies. N. Engl. J. Med. 305: 533-538. 
2. Long, M.W., L. L. Gragowski, C. H. Heffner \& L. A. Boxer. 1985. Phorbol diesters stimulate the development of an early murine progenitor cell. J. Clin. Invest. 76: 431-438.

3. MAZUR, E. M., R. HoffMAN \& E. BRUNO. 1981. Regulation of human megakaryocytopoiesis. J. Clin. Invest. 68: 733-741.

4. Mazur, E. M., R. Hoffman, J. Chasis, S. Meroles \& E. Bruno. 1981. Immunofluorescent identification of human megakaryocytic colonies using an antiplatelet glycoprotein antiserum. Blood 57: 277-286.

5. Vainchenkek, N., W. J. Guzchard \& J. Breton-Gorius. 1968. Growth of human megakaryocyte colonies in culture from fetal, neonatal and adult peripheral blood cells: An ultrastructural analysis. Blood Cells 5: 25-31.

6. WILlIAMS, N. \& H. JACKSON. 1968. Regulation of proliferation of murine megakaryocyte progenitor cells by cell cycle. Blood 52: $163-168$.

7. Williams, N., H. M. JackSON, R. R. EgER \& M. W. Long. 1981. The separate roles of factors in murine megakaryocyte colony formation. In Megakaryocyte Biology and Precursors: Cloning and Cellular Properties. B. L. Evatt, R. F. Levine \& N. Williams, Eds.: 69-75. North-Holland. Amsterdam.

8. Williams, N. \& R. Levine. 1982. The origin, development and regulation of megakaryocytes. Br. J. Haematol. 52: 173-180.

9. Williams, N., H. Jackson, A. P. C. Sherida, M. J. Murphy, JR., A. Elste \& M. A. S. MOORE. 1978. Regulation of megakaryocytopoiesis in long-term murine bone marrow cultures. Blood Cells 5: $43-48$.

10. Williams, N. \& H. JACKSON. 1982. Kinetic analysis of megakaryocyte numbers and ploidy levels in developing colonies from mouse bone marrow cells. Cell Tissue Kinet. 15: 483494.

11. Williams, N., R. R. Eger, H. M. Jackson \& D. J. Nelson. 1982. Two-factor requirement for murine megakaryocyte colony formation. J. Cell. Physiol. 110: 101-104.

12. Williams, N., H. Jackson, P. RalPh \& I. Nakoinz. 1981. Cell interactions influencing murine marrow megakaryocytes: Nature of the potentiator cell in bone marrow. Blood 57: $157-160$.

13. LoNG, M.W. \& N. WII.LIAMs. 1982. Immature megakaryocytes in the mouse: Physical characteristics, cell cycle status and in vitro responsiveness to thrombopoietic stimulatory factor. Blood 59: 569-757.

14. LoNG, M.W. \& N. WILliams. 1982. Differences in the regulation of megakaryocytopoiesis in the murine bone marrow and spleen. Leukemia Res. 6: 721-728.

15. Long, M. W., N. Whllams \& T. P. McDonald. 1982. Immature megakaryocytes in the mouse: In vitro relationship to megakaryocyte progenitor cells and mature megakaryocytes. J. Cell. Physiol. 112: 339-344.

16. Rabeli.ino, E. M., R. B. Levene, L. L. K. Leung \& R. L. Nachman. 1981. Human megakaryocytes. II. Expression of platelet proteins in early marrow megakaryocytes. J. Exp Med. 154: 88-100.

17. Rabellino, E. M., R. L. Nachman, N. Williams, R. J. Winchester \& G. D. Ross. 1974. Human megakaryocytes. I. Characterization of membrane and cytoplasmic components of isolated marrow megakaryocytes. J. Exp. Med. 149: 1273-1287.

18. Long, M. W. \& N. WiLliams. 1981. Immature megakaryocytes in the mouse: Morphology and quantification by acetylcholinesterase staining. Blood 58: 1032-1039.

19. Hoffman, R., H. H. Yang, E. Bruno \& J. E. Straneva. 1985. Purification and partial characterization of a megakaryocyte colony-stimulating factor from human plasma. J. Clin. Invest. 75: $1174-1182$.

20. Long, M. W., J. E. Smolen, P. Szczepanski \& L. A. Boxer. 1984. Role of phorbol diesters in in vitro murine megakaryocyte colony formation. J. Clin. Invest. 74: 1686-1692.

21. Long, M. W., R. Hutchinson, C. Heffner \& L. Gragowski. 1985. Human megakaryocyte colony development in vitro requires two synergistic activities. Clin. Res. 33: 347a.

22. Ihle, J. H., J. Keller, L. Henderson, F. Klein \& E. Pai.aszynski. 1982. Procedures for the purification of interleukin-3 to homogeneity. J. Immunol. 129: 2431-2436.

23. LeE, J. C., A. J. HAPEl \& J. N. Ihle. 1982. Constitutive production of a unique lymphokine (IL-3) by the Wehi-3 cell. J. Immunol. 128: 2393-2398.

24. Prystowsky, M. B., G. Otten, M. Naujokas, J. Vardiman, J. N. Ihle, E. Goldwasser 
\& F. W. FiTCH. 1984. Multiple hemopoietic lineages are found after stimulation of mouse bone marrow precursor cells with interleukin-3. Am. J. Pathol. 117: 171-179.

25. Goodman, J. W., E. A. Hall, K. L. Miller \& S. G. Shinpock. 1985. Interleukin-3 promotes erythroid burst formation in "serum-free" cultures without detectable erythropoietin. Proc. Natl. Acad. Sci. USA 82: 3291-3295.

26. Quesenberry, P. J., J. N. Ihle \& E. MCGrath. 1985. The effect of interleukin-3 and GM-CSA-2 on megakaryocyte and myeloid clonal colony formation. Blood 65: 214-217.

27. Long, M. W., C. H. HefFner \& L. L. Gragowski. 1986. In vitro differences in responsiveness of early (BFU-Mk) and late (CFU-Mk) murine megakaryocyte progenitor cells. In Megakaryocyte Development and Function: 179-186. Alan R. Liss. New York, NY.

28. Rovera, J. T. J. O'Brien \& L. Diamond. 1979. Induction of differentiation in human promyelocytic leukemia cells by tumor promoters. Science 204: 868-870.

29. END, D., N. Tolson, M. Y. YU \& G. GuRoF. 1982. Effects of 12-O-tetradecanoyl phorbol13-acetate on rat pheochromocytoma (PC 12) cells: Interactions with epidermal growth factor and nerve growth factor. J. Cell. Physiol. 111: 140-148.

30. Stuart, R. K. \& J. A. Hamilton. 1980. Tumor-promoting phorbol esters stimulate hematopoietic colony formation in vitro. Science 208: 402-404.

31. Stuart, R. K., L. L. Sensendrenner, R. K. Shadduck, A. Waheed \& C. Caramatti. 1983. Phorbol ester-stimulated murine myelopoiesis. Role of colony-stimulating factors. J. Cell. Physiol. 117: 30-38.

32. Stuart, R. K., J. A. Hamition, L. L. Sensendrenner \& M. A. S. Moore. 1981. Regulation of myelopoiesis in vitro: Partial replacement of colony-stimulating factors by tumorpromoting phorbol esters. Blood 57: 1032-1042.

33. Griffin, J. D., P. Lakcom \& D. W. KufE. 1985. TPA induces differentiation of purified human myeloblasts in the absence of proliferation. Exp. Hematol. 13: 1025-1032.

34. Fibach, E., P. A. Marks \& R. A. Rifkind. 1980. Tumor promoters enhance myeloid and erythroid colony formation by normal mouse hematopoietic cells. Proc. Natl. Acad. Sci. USA 77: $4152-4155$.

35. Fibach, E., R. Gambart, P. A. Shaw, G. Manaltis, R. C. Reuben, S. Shigeru, R. A. RJFKIND \& P. MARKS. 1979. Tumor promuter-mediated inhibition of cell differentiation: Suppression of the expression of erythroid functions in murine erythroleukemia cells. Proc. Natl. Acad. Sci. USA 70: 1906-1910.

36. Messner, H. A., N. Jamal \& C. IZaguirre. 1982. The growth of large megakaryocyte colonies from human bone marrow. J. Cell. Physiol. Suppl. 1: 45-51.

37. Solberg, L. A., JR., N. Jamal \& H. A. Messnfr. 1985. Characterization of human megakaryocytic colony formation in human plasma. J. Cell. Physiol. 124: 67-74.

38. Kimura, H., S. A. Burstein, D. Thorning, J. S. Powell, L. A. Harker, P. J. Fialkow \& J. W. ADAMSON. 1984. Human megakaryocytic progenitors (CFU-M) assayed in methylcellulose: Physical characteristics and requirements for growth. J. Cell. Physiol. 118: 87-96.

39. Gewirtz, A. M., E. Bruno, J. Elwel. \& R. Hoffman. 1983. In vitro studies of megakaryocytopoiesis in thrombocytotic disorders of man. Blood 61: 384-389.

40. LONG, M. W. \& C. H. HefFNer. 1988. Detection of human megakaryocyte antigens by solid-phase radioimmunoassay. Exp. Hematol. 16: 62-70.

41. Hsu, S. M., L. Raine \& H. FAnger. 1981. The use of avidin-biotin-peroxide complex (ABC) in immunoperoxidase technique-a comparison between $\mathrm{ABC}$ and unlabeled antibody (PAP) procedures. J. Histochem. Cytochem. 29: 577-580.

42. HARKER, L.A. \& C. N. FinCh. 1969. Thrombokinetics in man. J. Clin. Invest. 48: 963-974

43. Odel.., T. T. \& C. SHELTON. 1979. Increasing stimulation of megakaryocytopoiesis with decreasing platelet count. Proc. Soc. Exp. Biol. Med. 161: 531-533.

44. Odell, T. T., J. R. MurPhy \& C. W. JaCkson. 1976. Stimulation of megakaryocytopoiesis by acute thrombocytopenia in rats. Blood 48: 765-775.

45. Odell, T. T., C. W. Jackson, T. J. Friday \& D. E. Cimarsha. 1969. Effects of thrombocytopenia on megakaryocytopoiesis. J. Haematol. 17: 91-101.

46. EBbe, S. 1976. Biology of Megakaryocytes. In Progress in Hemostasis and Thrombosis, Vol. III. T. H. SPaet, Ed.: 211-229. Grune \& Stratton. New York, NY

47. Harker, L. A. 1968. Kinetics of thrombopoiesis. J. Clin. Invest. 47: 458-465. 
48. Odell, T. T., C. W. Jackson, T. J. Friday \& D. E. Charsha. 1969. Effects of thrombocytopenia on megakaryocytopoiesis. Br. J. Maematol. 17: 91-101.

49. Tayrien, G. \& R. D. Rosenberg. 1987. Purification and properties of a megakaryocyte stimulatory factor present both in the serum-free conditioned medium of human embryonic kidney cells and in thrombocytopenic plasma. J. Biol. Chem. 262: 3262-3268.

50. Greenberg, S. M., D. J. Kuter \& R. D. Rosenberg. 1987. In vitro stimulation of megakaryocyte maturation by megakaryocyte stimulatory factor. J. Biol. Chem. 262: 3269-3277. 